\title{
Dopamine Transporter Genotype Is Associated with a Lateralized Resistance to Distraction during Attention Selection
}

\author{
DDaniel P. Newman, ${ }^{1}$ Tarrant D.R. Cummins, ${ }^{1}$ Janette H.S. Tong, ${ }^{1}$ Beth P. Johnson, ${ }^{1}$ Hayley Pickering, ${ }^{1}$ \\ (Deter Fanning, ${ }^{1}$ Joseph Wagner, ${ }^{2}$ Jack T.T. Goodrich, ${ }^{2}$ Ziarih Hawi, ${ }^{1}$ Christopher D. Chambers, ${ }^{3}$ and \\ Mark A. Bellgrove ${ }^{1,2}$ \\ ${ }^{1}$ Monash University, School of Psychological Sciences, Clayton, Victoria 3800, Australia, ${ }^{2}$ The University of Queensland, Queensland Brain Institute and \\ School of Psychology, Brisbane, Queensland 4072, Australia, and ${ }^{3}$ Cardiff University, School of Psychology, Cardiff CF10 3AT, United Kingdom
}

\begin{abstract}
Although lateral asymmetries in orienting behavior are evident across species and have been linked to interhemispheric asymmetries in dopamine signaling, the relative contribution of attentional versus motoric processes remains unclear. Here we took a cognitive genetic approach to adjudicate between roles for dopamine in attentional versus response selection. A sample of nonclinical adult humans $(N=$ 518) performed three cognitive tasks (spatial attentional competition, spatial cueing, and flanker tasks) that varied in the degree to which they required participants to resolve attentional or response competition. All participants were genotyped for two putatively functional tandem repeat polymorphisms of the dopamine transporter gene (DAT1; SLC6A3), which are argued to influence the level of available synaptic dopamine and confer risk to disorders of inattention. DAT1 genotype modulated the task-specific effects of the various taskirrelevant stimuli across both the spatial competition and spatial cueing but not flanker tasks. Specifically, compared with individuals carrying one or two copies of the 10-repeat DAT1 allele, individuals without this allele demonstrated an immunity to distraction, such that response times were unaffected by increases in the number of distractor stimuli, particularly when these were presented predominantly in the left hemifield. All three genotype groups exhibited uniform costs of resolving leftward response selection in a standard flanker task. None of these significant effects could be explained by speed-accuracy trade-offs, suggesting that participants without the 10-repeat allele of the DAT1 tandem repeat polymorphism possess an enhanced attentional ability to suppress task-irrelevant stimuli in the left hemifield.
\end{abstract}

Key words: DAT1; dopamine; genetics; individual differences; selective attention; spatial attention

\section{Introduction}

Convergent evidence from human and animal pharmacology shows that cholinergic (Voytko et al., 1994; Witte et al., 1997) and noradrenergic (Coull et al., 2001) mechanisms modulate behavioral and neural indices of selective attention. Although pharmacological evidence also supports a role for dopamine in selective attention (Clark et al., 1989), dissociating its effects on response selection versus attentional selection has proven controversial (Ward and Brown, 1996). Here we show that DNA variation in a putatively functional dopamine polymorphism is associated with

Received June 7, 2014; revised Sept. 18, 2014; accepted 0ct. 12, 2014.

Author contributions: C.D.C. and M.A.B. designed research; D.P.N., T.D.R.C., J.H.S.T., B.P.J., H.P., P.F., J.W., J.T.T.G., Z.H., C.D.C., and M.A.B. performed research; T.D.R.C., J.H.S.T., and Z.H. contributed unpublished reagents/ analytic tools; D.P.N., C.D.C., and M.A.B. analyzed data; D.P.N., C.D.C., and M.A.B. wrote the paper.

This work was supported by a National Alliance for Research on Schizophrenia and Depression Young Investigator Grant (to M.A.B.), a project grant (569533) from the National Health and Medical Research Council (NHMRC) of Australia (to M.A.B.), and an NHMRC Career Development Award (569631; to M.A.B.). M.A.B. is currently supported by a Future Fellowship from the Australian Research Council of Australia (FT130101488).

The authors thank Natalia Lukito and Lynn Tan for data collection.

Correspondence should be addressed to Prof. Mark A. Bellgrove, School of Psychological Sciences, Monash University, Melbourne, VIC 3800, Australia. E-mail: mark.bellgrove@monash.edu.

DOI:10.1523/JNEUROSCI.2327-14.2014

Copyright $\odot 2014$ the authors $\quad 0270-6474 / 14 / 3315743-08 \$ 15.00 / 0$ enhanced attentional selection, particularly for stimuli presented within the left hemifield.

Damage to one cerebral hemisphere can induce hemispatial neglect where responses to contralesional stimuli are impaired. In humans, left neglect after right-hemisphere damage is more frequent and severe than right neglect after left-hemisphere damage (Driver and Mattingley, 1998). Consistent with animal studies that report spatial inattention after ascending dopaminergic pathway lesions (Iversen, 1984), dopamine agonists reduce the extent of spatial inattention in neurological (Fleet et al., 1987; Gorgoraptis et al., 2012) and psychiatric disorders (Maruff et al., 1995). Ward and Brown (1996), however, examined the impact of unilateral dopamine-depleting lesions on covert orienting in rats. Although unilateral dopamine depletion slowed response times contralateral to the lesion, responses to validly and invalidly cued targets did not differ. These authors concluded that striatal dopamine influences response but not selective attention processes.

A few studies have reported that DNA polymorphisms of the dopamine transporter gene (DAT1) are associated with asymmetries of spatial attention (Bellgrove et al., 2005, 2007, 2008, 2009; Newman et al., 2012; Zozulinsky et al., 2014). The 10-repeat allele of a variable number of tandem repeat (VNTR) polymorphism 
Table 1. Genotype specific demographics and associated significance tests

\begin{tabular}{|c|c|c|c|c|c|c|c|c|c|c|c|c|}
\hline & \multicolumn{4}{|c|}{ DAT13'UTR VNTR group } & \multicolumn{4}{|c|}{ DAT1 intron 8 VNTR group } & \multicolumn{4}{|c|}{ DAT1 10/6 haplotype group } \\
\hline & $\begin{array}{l}\text { Zero 10-repeats } \\
(n=36)\end{array}$ & $\begin{array}{l}\text { One 10-repeat } \\
(n=182)\end{array}$ & $\begin{array}{l}\text { Two 10-repeats } \\
(n=300)\end{array}$ & $\begin{array}{l}p \\
\text { value }\end{array}$ & $\begin{array}{l}\text { Zero 6-repeats } \\
(n=24)\end{array}$ & $\begin{array}{l}\text { One 6-repeat } \\
(n=167)\end{array}$ & $\begin{array}{l}\text { Two 6-repeats } \\
(n=336)\end{array}$ & $\begin{array}{l}p \\
\text { value }\end{array}$ & $\begin{array}{l}\text { Zero copies } \\
(n=43)\end{array}$ & $\begin{array}{l}\text { One copy } \\
(n=212)\end{array}$ & $\begin{array}{l}\text { Two copies } \\
(n=257)\end{array}$ & $\begin{array}{l}p \\
\text { value }\end{array}$ \\
\hline $\begin{array}{l}\text { CAARS score: } \\
\text { mean (SD) }\end{array}$ & $44.8(8.0)$ & $48.8(9.7)$ & $49(7.9)$ & 0.02 & $46.8(7.8)$ & $47.6(9)$ & $49.1(4.5)$ & 0.11 & $45.3(8.3)$ & $48.6(9.3)$ & $49.3(8.1)$ & 0.02 \\
\hline
\end{tabular}

within the $3^{\prime}$ untranslated region of DAT1 is an established susceptibility locus for attention deficit/hyperactivity disorder (ADHD). In vitro and human in vivo experiments suggest this variant is associated with increased expression of the transporter, which could reduce available synaptic dopamine (Heinz et al., 2000; Fuke et al., 2001; Cheon et al., 2005; VanNess et al., 2005; Brookes et al., 2006, 2007). Allelic variation within both this VNTR and an intron 8 VNTR has been associated with asymmetries of selective attention in children with and without ADHD (Bellgrove et al., 2007, 2009). Although Newman et al. (2012) reported an influence of the DAT1 3'UTR VNTR on lateralized target detections in nonclinical adults, participants were not required to select a target from among competing distractors, making it unclear whether the results merely reflect an asymmetry of response selection rather than a specific asymmetry of attentional selection.

Using three paradigms with varying requirements for resolving attentional competition versus response competition, we took a cognitive genetic approach to adjudicate between roles for dopamine in attentional versus response selection. If DAT1 genotype is associated with response selection mechanisms (lateralized or not), then global differences in speed should exist across tasks and DAT1 genotype. If, however, DAT1 genotype is linked to lateralized attentional selection, then DAT1 genotype should influence the suppression of task-irrelevant stimuli, particularly when distraction arises in the left hemifield.

\section{Materials and Methods}

Participants

Participants $(N=532)$ were recruited and tested at either the University of Queensland ( $n=379)$ or Monash University $(n=153)$ using identical procedures. A "cohort" factor was included as a covariate in all analyses to control for any subtle differences between testing locations. Healthy right-handed participants were included in the current analysis if they (1) completed all three tasks; (2) were of Caucasian background; (3) had completed the Cognitive Failures Questionnaire (CFQ) and Conners' Adult ADHD Rating Scales (CAARS), self-report scales that screened for subthreshold attention deficits; and (4) were successfully genotyped for the $3^{\prime}$-UTR and intron 8 VNTRs of the DAT1 gene. These selection criteria resulted in the inclusion of 518 (270 females and 248 males) of the 532 participants (see Table 1 for genotype-specific demographics).

\section{Cognitive tasks}

The three cognitive tasks (see Spatial competition, Spatial cueing, and Flanker tasks below) were presented in a dimly lit room on an $85 \mathrm{~Hz}$, $12 \times 16$ inch CRT monitor positioned at a viewing distance of $65 \mathrm{~cm}$ (stimulus visual angle, $3.6 \times 3.3^{\circ}$ ). Participants were instructed to horizontally align themselves with a fixation cross $(+)$ that was presented in the center of the screen and to make responses via a standard Dell QWERTY keyboard with USB connection. Although use of a keyboard for recording responses introduces some timing imprecision, empirical and modeling research shows that this additional error variability does not affect statistical outcomes for research designs such as that used here, where repeated-measures experimental comparisons are emphasized (Damian, 2010; Brand and Bradley, 2012). The tasks were presented in counterbalanced order across participants with optional rest breaks of $\leq 60$ s between each task block. Participants were always instructed to maintain fixation and were reminded that the tasks were easiest to perform when fixation was maintained. The task requirements are detailed below and the temporal parameters of each task can be seen in Figure 1.

Horizontal saccadic latency (the interval between the appearance of a target and the onset of eye movement) for healthy adults is typically 200-250 ms in "overlap paradigms" where the central fixation point remains visible up to and during the time the target appears (Yang et al., 2006), as was the case in the spatial competition and cueing tasks (Fig. $1 A, B)$. No eye-tracking data was collected. However, because the target was displayed in the spatial competition and spatial cueing tasks for only $150 \mathrm{~ms}$, which is less than typical horizontal saccadic latency, we assumed that participants did not have time to begin moving their eyes toward the target. In the spatial competition task (in which the largest lateralized DAT1 effect was observed), the participants had no prior information about the location of the forthcoming target and so had no information to elicit a pretarget fixation break. In the spatial cueing task, the cue-totarget duration (stimulus onset asynchrony) was $150 \mathrm{~ms}$, which is too brief for an eye movement toward the cue before target onset. Since the critical dependent measure in the spatial cueing task was the reorienting cost (from irrelevant cues), it is highly unlikely that eye movements could account for the lateralized effect on the spatial cueing task.

Spatial competition task. The spatial competition task began with the simultaneous presentation of a fixation cross and an array of 16 circular placeholders (white outlines) of $2 \mathrm{~cm}$ diameter that were arranged to form a global circle ( $15 \mathrm{~cm}$ diameter) on a black background. To challenge attentional selection mechanisms, targets appeared surrounded by either three or seven distractors, corresponding to total set sizes of four and eight, respectively. The target in each trial was an upright or inverted $\mathrm{T}$ and the distractors were plus signs $(+)$. The distractors were presented in either the same hemifield as the target (unilateral) or in both the same and the opposite hemifield (bilateral; Fig. $1 A$ shows a bilateral 8 condition). Equal numbers of each of these trial types were presented in pseudorandom order. During each trial, the target could appear within any circle (counterbalanced for left and right appearance across trials). Participants indicated the orientation of the target by using their right index and middle fingers, respectively, to press 1 for upright or 2 for inverted on the number pad of the keyboard. Stimuli arrays varied in regards to both the number and spatial layout of distractors. Participants were presented with three practice blocks of 32 trials followed by 8 test blocks of 40 trials (320 in total). Feedback (correct, incorrect) was given in practice blocks only.

The spatial competition task was devised to assay attentional selection. Although overall increases in response time with set size were expected, we were interested in testing the specific prediction that DAT1 genotype was linked to a lateralized attentional selection mechanism. This hypothesis was tested by comparing the "set-size effect" operationalized as the behavioral cost of additional distractors that were presented predominantly in the left or predominantly in the right hemifield.

Spatial cueing task. The spatial cueing task was an exogenous covert orienting task based on the cued target detection task of Posner et al. (1984), a paradigm that has been widely used to assess spatial attention. 


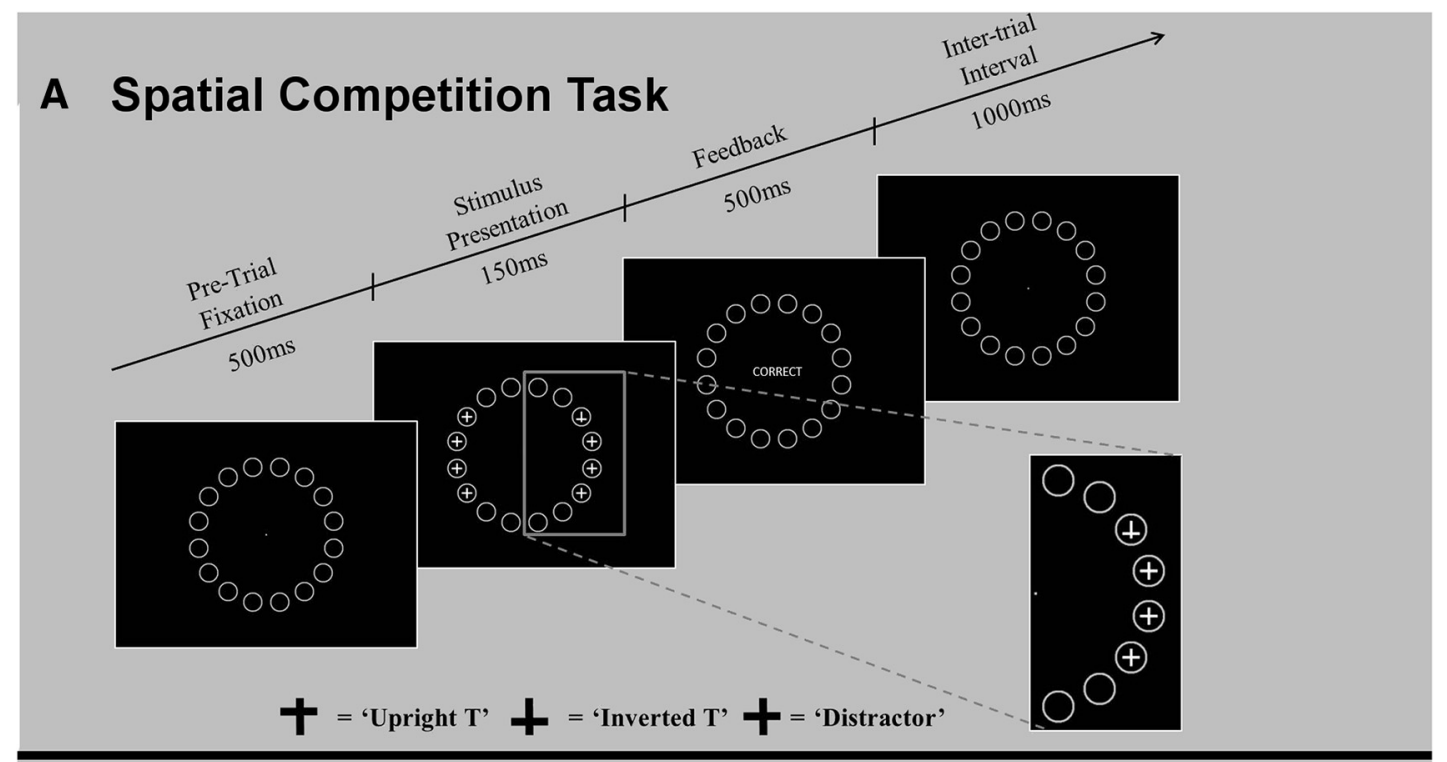

\section{B Spatial Cueing Task}

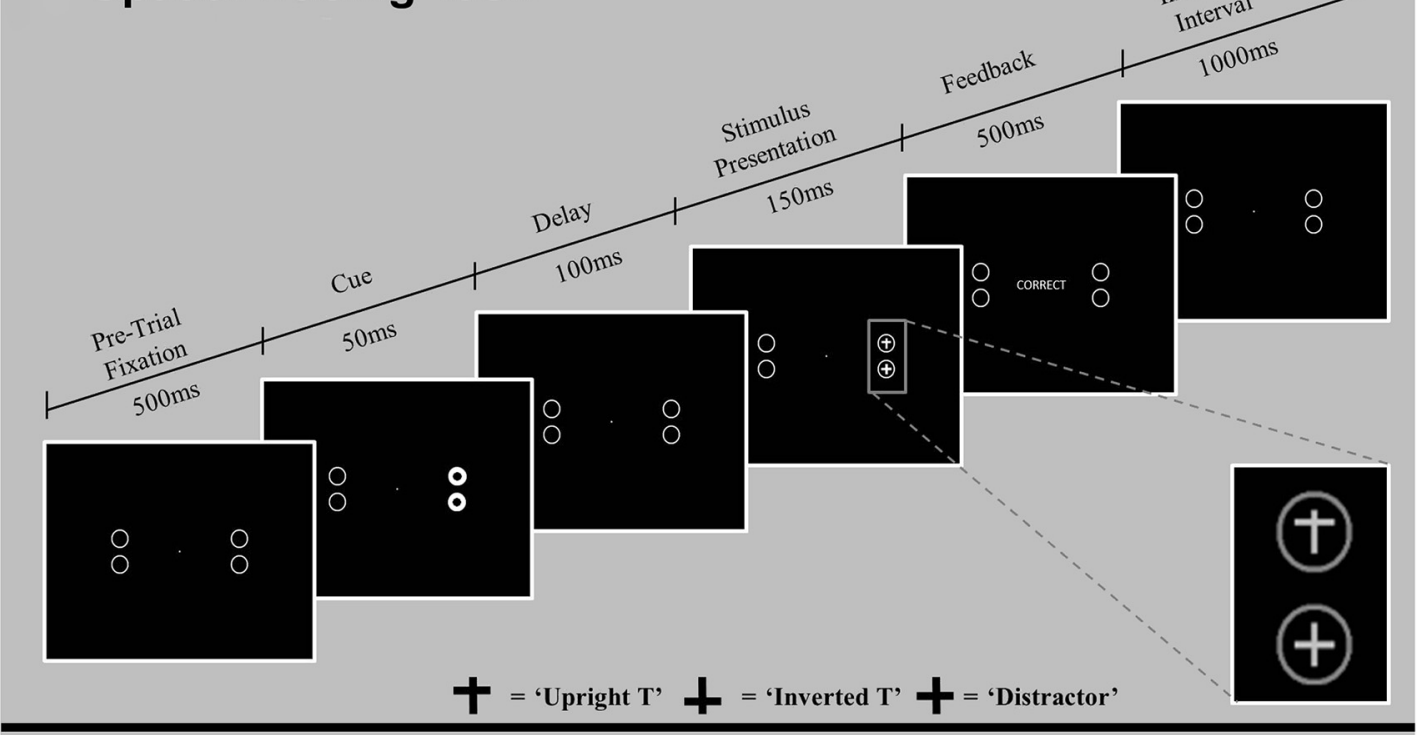

\section{Flanker Task}

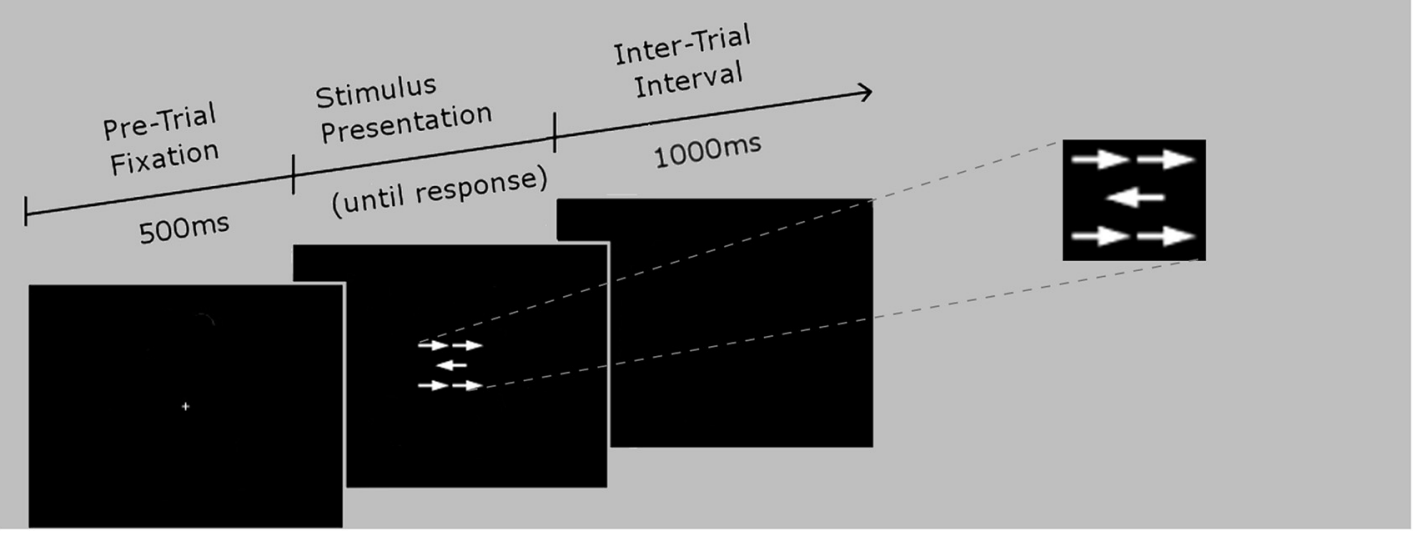

Figure 1. Task schematics of the spatial competition $(\boldsymbol{A})$, spatial cueing $(\boldsymbol{B})$, and flanker $(\boldsymbol{C})$ tasks used to probe attentional and response selection mechanisms. 
Covert orienting was encouraged by instructing participants to focus on a fixation cross that was present for the entire duration of each trial. After presenting the fixation cross alone, four circular placeholders (white outline) of $2 \mathrm{~cm}$ diameter each were displayed in a square arrangement against a black background. Cues consisted of a 100\% increase in luminance of some or all of the placeholders. Cues either appeared on the same side as the target (valid), the opposite side of the target (invalid), or gave no information regarding the spatial location of the upcoming target (neutral; all four placeholders "illuminated"; Fig. 1B). Equal numbers of each of these trial types were presented in pseudorandom order. In each trial, a single target (an upright or inverted T) appeared in one of the placeholders on a given side of the array (counterbalanced across trials for vertical arrangement and for occurrence on left and right). A distractor stimulus $(+)$ was presented at the other placeholder location within the same hemifield as the target. Participants were instructed to indicate the orientation of the target as per the competition task. Participants completed three practice blocks of 24 trials ( 72 in total) followed by eight test blocks of 36 trials (288 in total). For the spatial cueing task, two primary dependent measures were calculated: the behavioral cost of reorienting attention to the target from left or right cues ("reorienting cost": invalid vs bilateral), and the behavioral benefit of having oriented attention validly toward the target due to left or right cues ("orienting benefit": bilateral vs valid).

Flanker task. Participants completed a flanker task (Eriksen and Yeh, 1985; Eriksen and St James, 1986) comprising a single practice block of 36 trials and eight test blocks of 36 trials each (288 test trials in total). In each trial, participants made a directional response (left or right) to a central target arrow while ignoring four flanking distractors (Fig. 1C). Relative to the direction of the central target, the set of flankers provided information that was either congruent (same-facing arrows), incongruent (opposite-facing arrows), or neutral (lines without arrowheads). Equal numbers of each of these trial types were presented in pseudorandom order. Participants indicated the direction of the central arrow in each trial with their index fingers using the $\mathrm{Z}$ (left) and $\mathrm{M}$ (right) keys of the keyboard, with emphasis on both speed and accuracy. The flanker task was employed to challenge response competition and the critical measure was the behavioral cost of overcoming incongruent flankers ("incongruence cost": incongruent vs neutral) according to the flanker direction (left, right).

\section{Genotyping}

Saliva was collected from each participant for DNA extraction using Oragene DNA self-collection kits (DNAgenotek). DNA was extracted following the protocol provided by the supplier. Participants were genotyped for the $3^{\prime}$-UTR and intron 8 VNTRs of the DAT1 gene (SLC6A3). PCR amplification of the DAT1-3' UTR marker was performed using the following primers (50 ng each): forward, 5' -TGTGGTGTAGGGAACGGCCTGAG-3'; reverse, 5'-CTTCCTGGAGGTCACGGCTCAAGG-3'. The following PCR cycling protocol was adopted: initial denaturation at $94^{\circ} \mathrm{C}$ for $2 \mathrm{~min}$, followed by 45 cycles of denaturation at $94^{\circ} \mathrm{C}$ for $15 \mathrm{~s}$, annealing at $62^{\circ} \mathrm{C}$ for $15 \mathrm{~s}$, and extension at $72^{\circ} \mathrm{C}$ for $45 \mathrm{~s}$, with a final 5 min extension at $72^{\circ} \mathrm{C}$.

PCR of the DAT1-intron 8 VNTR was performed using the following primers (50 ng each): forward, 5'-CTCAGCTTCCTCATGTGCCT-3'; reverse, 5'-GCAGAAACAAGGAGGAGCAG-3'. The following PCR cycling protocol was adopted: initial denaturation at $98^{\circ} \mathrm{C}$ for $30 \mathrm{~s}$, followed by 35 cycles of denaturation at $98^{\circ} \mathrm{C}$ for $10 \mathrm{~s}$, annealing at $65^{\circ} \mathrm{C}$ for $30 \mathrm{~s}$, and extension at $72^{\circ} \mathrm{C}$ for $15 \mathrm{~s}$. A final 10 min extension at $72^{\circ} \mathrm{C}$ was also added. All amplification products were visualized on $1.5 \%$ agarose gels. For the $3^{\prime}$-UTR and intron 8 DAT1 VNTRs, grouping variables were coded based on the presence of the functional 10-repeat and 6-repeat alleles, respectively. The frequency of the 10-repeat allele of the $3^{\prime}$-UTR VNTR was $75 \%$, with 36,182 , and 300 individuals possessing zero, one, or two copies of the 10-repeat, respectively. The frequency of the 6-repeat allele of the intron 8 VNTR was $80 \%$, with 24,167 , and 336 individuals possessing zero, one, or two copies of the 6-repeat, respectively. All genotypes for both VNTRs were in Hardy-Weinberg equilibrium. Haplotypes comprising the 10-repeat and 6-repeat alleles from each marker were derived using Phase version 2.1 (Stephens et al., 2001; Stephens and
Donnelly, 2003). Possession of the 10/6 DAT1 haplotype is a risk factor for ADHD and has been linked to asymmetries of spatial attention in children with and without ADHD (Bellgrove et al., 2007, 2009). For statistical analysis, individuals were classified as possessing zero, one, or two copies of the 10-repeat or 6-repeat alleles of the VNTRs or of the 10/6 DAT1 haplotype.

\section{Results}

\section{Influence of DAT1 gene variants on demographic and} self-report variables

A one-way ANOVA revealed a significant effect of DAT1 3'-UTR genotype group on mean CAARS ADHD index scores $\left(F_{(2,362)}=\right.$ $2.6, p=0.02)$, which is consistent with past reports of an association between increasing copies of the 10-repeat DAT1 allele and ADHD-like behavior in both clinical and population-based samples (Cook et al., 1995; Cornish et al., 2005). To optimize the sensitivity of the genetic comparisons, all analyses were controlled simultaneously for potential (subthreshold) group differences in variables that could potentially influence either attention or response selection. These covariates included gender, CAARS ADHD index, and everyday inattention assessed using the CFQ and recruitment cohort (University of Queensland, Monash University). Descriptive statistics concerning these covariates as a function of all genetic markers are seen in Table 1 . We note that we reran the analysis for our key findings without the use of covariates, in line with the recommendation of Simmons et al. (2011). This revealed that the key effects reported below hold when covariates are not used in the analysis. This reanalysis can be accessed on-line.

\section{Omnibus task-component analyses}

An initial $3 \times 4 \times 2$ mixed ANCOVA of reaction time $(\mathrm{RT})$ included the between-subjects factor of DAT1 3'-UTR genotype group (zero, one, or two 10-repeat alleles) and within-subjects factors of Task-Component (set-size effect, reorienting cost, orienting benefit, incongruence cost) and Task-Irrelevant Stimulus Laterality (left, right). This analysis revealed a significant DAT1 $\times$ Stimulus Laterality interaction $\left(F_{(2,511)}=4.9, p=0.008\right.$, $\left.\eta_{p}^{2}=0.02\right)$ resembling to some extent previous reports of an association between DAT1 genotype and a spatial bias in response to lateralized stimuli (Newman et al., 2012). Critically, however, this was qualified by a significant three-way interaction of DAT $1 \times$ Task-Component $\times$ Stimulus Laterality $\left(F_{(6,1533)}=\right.$ 2.6, $\left.p=0.017, \eta_{p}^{2}=0.01\right)$ indicating that DAT1 genotype modulated task-specific effects of the various task-irrelevant stimuli on RT. The same analysis for percentage errors (\%Errors) revealed no DAT $1 \times$ Task-Component $\times$ Stimulus Laterality interaction $\left(F_{(6,1533)}=0.59, p=0.74\right)$.

Separate task-specific analyses of RT were undertaken below to identify the source of the significant three-way interaction. For completeness, and to ensure that any observed effects on RT were not due to speed-accuracy trade-offs, the same follow-up analyses were also undertaken on \%Errors. We note that the equivalent analyses focusing on the influence of the 6-repeat allele of the intron 8 DAT1 VNTR and the 10/6 haplotype derived from both DAT1 VNTRs were nonsignificant $\left(\mathrm{RT}_{\text {introns }}: F_{(6,1560)}=0.76\right.$, $p=0.61 ; \mathrm{RT}_{\text {haplotype }}: F_{(6,1166.4)}=2.0, p=0.08 ; \%$ Errors $_{\text {intron } 8}$ : $F_{(6,1560)}=0.41, p=0.88 ; \%$ Errors $_{\text {haplotype }}: F_{(6,1515)}=0.90, p=$ $0.49)$. For this reason all further analysis focused on the association with the 10-repeat allele of the DAT1 3 '-UTR VNTR.

\section{Spatial competition task}

To identify the source of the significant interaction between DAT1 3 '-UTR $\times$ Task-Component $\times$ Stimulus Laterality re- 
A

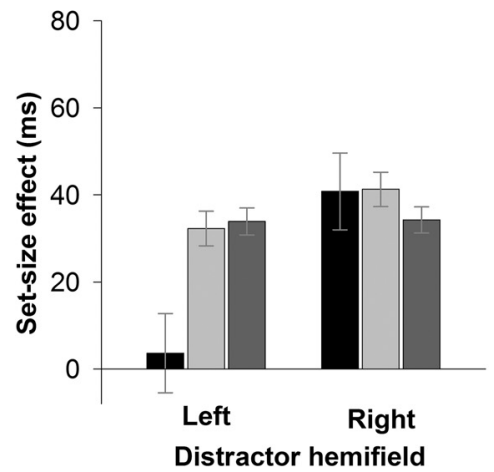

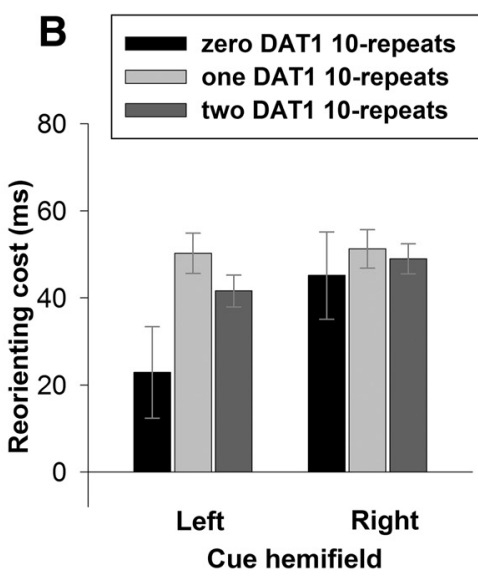

Figure 2. $\quad \boldsymbol{A}-\boldsymbol{C}$, Interactions of DAT1 3'UTR VNTR genotype as a function of task-irrelevant stimulus laterality (left, right) for each task component in each of the spatial competition $(\boldsymbol{A})$, spatial cueing $(\boldsymbol{B})$, and flanker $(\boldsymbol{C})$ tasks. $\boldsymbol{A}$, Set-size effect (RT, ms) during the spatial competition task was significantly reduced in the zero 10 -repeat DAT1 group for left hemifield distractors. $\boldsymbol{B}$, The reorienting cost (RT, ms) engendered by left cues was reduced in the zero 10-repeat repeat DAT1 group. $C$, There was no effect of DAT1 genotype on response selection as measured by flanker task incongruence cost (RT, ms). Error bars are $\pm 1 \mathrm{SE}$.

ported in the omnibus analysis above, we first analyzed the spatial competition RT data, testing the influence of DAT1 genotype as a function of distractor hemifield on the critical measure of set-size effect (set-size 8 minus set-size 4). The DAT1 $\times$ Distractor Hemifield (predominantly left vs predominantly right hemifield) $\times$ Display Type (unilateral, bilateral) ANCOVA revealed a significant interaction of DAT1 genotype and distractor hemifield for the set-size effect $\left(F_{(2,511)}=5.5, p=0.004, \eta_{p}^{2}=0.02\right)$ and no other significant effect involving DAT1. For right hemifield distractors, the set-size effect did not differ significantly between the zero 10-repeat (mean, $40.8 \mathrm{~ms}$; SE, $8.8 \mathrm{~ms}$ ), one 10-repeat (mean, $41.3 \mathrm{~ms}$; SE, $3.9 \mathrm{~ms}$ ), and two DAT1 10-repeat groups (mean, 34.3 ms; SE, $3.0 \mathrm{~ms}$; all $p>0.49$ ). For left distractors, however, the set-size effect was significantly smaller in the zero 10-repeat group (mean, $3.6 \mathrm{~ms}$; SE, $9.1 \mathrm{~ms}$ ) than in both the one 10-repeat [mean, $32.3 \mathrm{~ms}$; SE, $4.0 \mathrm{~ms} ; p=0.004$, Cohen's $d(d)=0.53$ ] and two DAT1 10-repeat groups (mean, $34 \mathrm{~ms}$; SE, $3.1 \mathrm{~ms} ; p=0.005$, $d=0.56)$, which did not differ significantly from each other $(p=$ 0.75; Fig. 2A). Bootstrapping (10,000 samples) the sampling distribution of the simple effect of DAT1 genotype for left hemifield distractors confirmed that the set-size effect was significantly smaller in the zero 10-repeat group than in both the one 10repeat $(95 \%$ CI for difference, -46.5 to -9.5 ) and two DAT1 10 -repeat groups ( $95 \% \mathrm{CI}$ for difference, -46.9 to -12.1 ). These analyses of the set-size effect on RT thus indicate an enhanced resistance to left-sided distraction in participants with zero copies of the DAT1 10-repeat allele. Figures depicting absolute RT values for the spatial competition task, as opposed to the set-size effect, can be accessed on-line.

To confirm that the resistance to distraction in the zero DAT1 10-repeat group reflected a true performance benefit rather than a speed-accuracy trade-off, the same analysis of set-size effects was undertaken on \%Errors (Fig. 2B). The set-size effects in all conditions were consistently between 11 and $13 \%$ and did not differ significantly between the DAT1 groups for left or right distractors (all $p>0.27$ ). Nevertheless, it is notable that for lefthemifield distractors, the zero DAT1 10-repeat group had a numerically reduced (rather than increased) set-size effect (mean, $11.5 \%$; SE, $1.3 \%$ ) relative to the one 10 -repeat (mean, $12.0 \%$; SE, $0.6 \%$ ) and two DAT1 10-repeat groups (mean, $12.6 \%$; SE, $0.5 \%$ ). Thus, the resistance to left hemifield distraction for the zero DAT1 10-repeat group shown in the RT data cannot be explained by a speed-accuracy trade-off. Although there was no additional interaction with the factor of Display Type (unilateral, bilateral), for transparency the above DAT1 by distractor hemifield interaction is plotted separately for unilateral and bilateral displays and can be accessed on-line.

\section{Spatial cueing task}

Again, to identify the locus of the significant omnibus effect for $\mathrm{RT}$, analyses of the spatial cueing data considered separately the reorienting costs and orienting benefits afforded by left and right cues, relative to the bilateral cueing condition. Figures depicting absolute RT values for the cueing task, as opposed to the reorienting costs and orienting benefits, can be accessed on-line.

\section{Reorienting cost}

For RT (Fig. 2B), the average reorienting cost was generally lower in the zero DAT1 10-repeat group compared with the other groups. Specifically, the cost of reorienting from left cues in the zero 10-repeat group (mean, $22.9 \mathrm{~ms}$; SE, $10.5 \mathrm{~ms}$ ) was significantly reduced relative to the one 10-repeat group (mean, $50.2 \mathrm{~ms} ; \mathrm{SE}, 4.6 \mathrm{~ms} ; p=0.018, d=0.31$ ) and tended to differ in the same direction from the two 10-repeat group (mean, $41.6 \mathrm{~ms}$; SE, $3.6 \mathrm{~ms} ; p=0.09, d=0.30)$. There were no DAT genotype effects for right cues (all $p>0.5)$. Bootstrapping (10,000 samples) the sampling distribution of the simple effect of DAT1 genotype confirmed that the cost of reorienting from invalid left hemifield cues was significantly reduced for the zero 10-repeat group relative to the one 10 -repeat group (95\% CI for difference, -45.8 to -9.1 ) and two DAT1 10-repeat groups (95\% CI for difference, -35.6 to $-1.6)$.

The reorienting cost for \%Errors did not differ as a function of DAT1 genotype for left cues (all $p>0.1$ ). For the right cues, the reorienting cost in the zero 10-repeat group (mean, $-0.85 \%$; SE, $1.3 \%)$ was significantly reduced relative to those with one 10 repeat (mean, $2.3 \%$; $\mathrm{SE}=0.56 \% ; p=0.026, d=0.41$ ), while the difference between one and two 10-repeat (mean, 1.3\%; SE, $0.44 \%)$ groups did not differ $(p=0.182)$.

\section{Orienting benefit}

There was no significant effect of DAT1 3'UTR genotype on orienting benefit for either RT or \%Errors for either left or right cues. 


\section{Flanker task}

Analysis of both RT and \%Errors for the critical measure of incongruence cost for left and right distractors revealed no significant effects involving the DAT1 3'UTR VNTR (Fig. 2C). Figures depicting absolute RT values for the flanker task, as opposed to the incongruence cost, can be accessed on-line.

\section{Additional control analysis}

These results suggest that subjects with zero copies of the 10repeat allele of the DAT1 3'UTR VNTR benefit from enhanced attentional selection, being particularly efficient at suppressing task-irrelevant stimuli presented in the left hemifield. To what extent, however, might this effect result from a generalized impediment of stimulus processing in the left hemifield? In other words, might zero 10-repeat subjects also be impaired at detecting task-relevant stimuli in the left hemifield?

The analyses so far indicate that this is unlikely. If subjects were impaired at processing all stimuli in the left hemifield, then the cost of reorienting from right cues (and thus toward left targets) should be greater in the zero DAT1 10-repeat group compared with the other DAT1 genotype groups; however, the opposite trend was in fact observed (Fig. 2B). Nevertheless, to confirm directly that the observed suppressive effects were specific to task-irrelevant stimuli, two additional analyses were conducted.

First, in the spatial competition task, we compared performance for left targets between the three genotype groups, collapsing across all other conditions. Second, in the spatial cueing task, we compared performance for left targets on trials with bilateral (neutral) cues between the groups. In both cases, a nonspecific suppression of all stimuli in the left hemifield would predict slower responses in the zero DAT1 10-repeat group compared with the other genotype groups. On the other hand, an enhancement of attentional selection would predict either no difference or the opposite effect, namely, faster responses to task-relevant stimuli presented in the "enhanced" hemifield.

For the spatial competition task, a one-way ANCOVA of mean RT to left targets (additionally covarying for error rates to left targets) revealed a marginal main effect of DAT1 group $\left(F_{(2,511)}=2.6, p=0.076, \eta_{p}^{2}=0.010\right)$, driven by faster responses in the zero DAT1 10-repeat group (mean, $672 \mathrm{~ms}$; SE, $20 \mathrm{~ms}$ ) compared with the one 10-repeat (mean, $721 \mathrm{~ms}$; SE, $9 \mathrm{~ms} ; p=$ $0.028, d=0.41$ ) and two 10-repeat groups (mean, $719 \mathrm{~ms}$; SE, 7 $\mathrm{ms} ; p=0.029, d=0.39$ ). For the spatial cueing task, the same analysis for left targets on bilateral trials (again additionally covarying for error rates to left targets) revealed no significant main effect of DAT1 group $\left(F_{(2,511)}=2.06, p=0.129, \eta_{p}^{2}=0.008\right)$, but pairwise comparisons show the zero DAT1 10-repeat group had numerically faster responses (mean, $618 \mathrm{~ms}$; SE, $19 \mathrm{~ms}$ ) compared with both the one 10-repeat (mean, $657 \mathrm{~ms} ; \mathrm{SE}, 8 \mathrm{~ms} ; p=$ $0.06, d=0.35$ ) and two 10-repeat groups (mean, $658 \mathrm{~ms} ; \mathrm{SE}, 7$ $\mathrm{ms} ; p=0.045, d=0.36$ ). These findings thus rule out a generalized impairment of stimulus processing in the left hemifield and indicate that individuals who do not possess the 10-repeat allele of the DAT1 3'UTR VNTR demonstrate a selective enhancement in suppressing task-irrelevant stimuli, i.e., a left hemifield enhancement of attention.

\section{Discussion}

This study provides evidence that allelic variation in a common polymorphism of the dopamine transporter gene (DAT1) mediates aspects of attentional but not response selection. Compared with individuals carrying DAT1 variants that increase risk for psychiatric disorder (Brookes et al., 2006; Guindalini et al., 2006), individuals lacking this same variant displayed a remarkable resistance to task-irrelevant distracting stimuli when these appeared predominantly in the left hemifield. These results cannot be explained in terms of a speed-accuracy trade-off, a generalized impairment of stimulus processing in the left hemifield, or by a leftward bias in response selection. Instead, our study provides evidence of a genetically mediated enhancement in suppressing task-irrelevant distraction in the left hemifield.

The primary question under study here was whether genetic variation in the DAT1 gene was linked to a lateralized attentional but not response selection mechanism. To assay attentional selection, participants completed the spatial competition task (Fig. $1 A)$. When distractors were predominantly in the right hemifield, each of the DAT1 genotype groups displayed the expected set-size effect, with RT being slower at larger compared with smaller set sizes. This indicates invariance as a function of DAT1 genotype in the ability to reject distracting information arising predominantly from the right hemifield. In contrast, we observed a significant effect of DAT1 genotype on the set-size effect when distractors were presented predominantly in the left hemifield, regardless of whether the display type was unilateral or bilateral. This effect arose because those individuals without the 10-repeat DAT1 allele displayed a diminished set-size effect for displays with predominantly left-sided distractors. These results indicate that individuals without the 10-repeat DAT1 allele have a specific advantage in distinguishing relevant from irrelevant information in the left but not right hemifield.

We tested the generality of our DAT1-mediated attentional selection hypothesis using data from a Posner cueing task, in which the data were again coded according to the hemifield of the task-irrelevant stimulus (cue). We derived costs and benefits afforded by left and right cues relative to the bilateral cueing condition. In line with the data from the attentional competition task, we observed reduced reorienting costs from left cues in individuals without the 10-repeat DAT1 allele compared with individuals possessing this allele. These data accord with the hypothesis that task-irrelevant information presented in the left hemifield have diminished salience and therefore reduced potential to slow reorienting in individuals who do not possess the 10 -repeat DAT1 allele. In contrast to the effects of DAT1 genotype on the selective attention tasks described above, no significant effects of DAT1 genotype were observed for the flanker task. All DAT1 genotype groups displayed a pronounced flanker incongruence cost, regardless of whether the flanking stimulus primed a left compared with right motor response. Taken together, the results of this study suggest that DAT1 genotype mediates a lateralized attentional, but not response, selection mechanism.

So what might be the mechanism by which DNA variation in a dopamine gene differentially impacts attentional selection mechanisms between the hemifields? A large amount of data now suggests that asymmetries within the dopamine system may give rise to lateral differences in behavior. First, it is well established that animals exhibit spontaneous orienting behavior that is consistent within the individual and is linked to asymmetries in striatal dopamine. For example, Glick and Shapiro (1985) have shown that animals spontaneously orient contralaterally to the striatum with higher dopamine levels. Furthermore, Gordon et al. (1994) demonstrated that orienting behavior in rodents was linked to lower dopamine transporter (DAT) reuptake, and thus higher levels of synaptic dopamine, in the striatum contralateral to the preferred orienting direction. Second, a number of studies in humans have demonstrated that dopamine agonists, including 
methylphenidate, which inhibits reuptake of dopamine via DAT, remediate the pathological inattention to left space that is seen in patients with hemispatial neglect and children with ADHD (Fleet et al., 1987; Grujic et al., 1998; Sheppard et al., 1999; Gorgoraptis et al., 2012; Silk et al., 2014). Third, Tomer et al. (2012) have recently reported that perceptual asymmetries measured using a free-viewing perceptual task (the Greyscales Task) in healthy adult participants were tightly correlated with asymmetric binding of dopamine D2 receptors in the striatum, such that spatial bias was directed contralaterally to the hemisphere with higher D2 receptor binding. These studies clearly establish a link between perceptual biases and striatal dopamine asymmetries, with relatively higher available dopamine in one hemisphere promoting directed attention to the contralateral hemifield.

In accordance with the above, a number of previous studies have reported associations between the DAT1 10-repeat allele and perceptual biases, most typically measured using clinical tests of hemispatial neglect, such as the Landmark Task. Bellgrove et al. $(2005,2008)$ asked children with ADHD to perform the Landmark Task, in which they were required to judge which end of a prebisected line was shorter. Children with ADHD carrying two copies of the DAT1 10-repeat allele displayed a neglect-like perceptual bias to right, indicative of weaker right hemisphere function. In contrast, ADHD individuals heterozygous for the DAT1 10-repeat allele displayed a perceptual bias to the left. Further, Newman et al. (2012) observed a perceptual advantage for the left hemifield in individuals heterozygous for the DAT1 10-repeat allele, with faster detection of targets in the left compared with the right hemifield. Homozygosity for the 10-repeat allele was associated with a relative loss of this leftward advantage. This association between the 10-repeat DAT1 allele and a rightward spatial bias was recently replicated in a sample of healthy adults by Zozulinsky et al. (2014).

Both in vitro gene reporter and human in vivo brain imaging work provide evidence that the 10-repeat allele of the DAT1 3'UTR may be associated with increased DAT expression/binding (Heinz et al., 2000; Fuke et al., 2001; Cheon et al., 2005; VanNess et al., 2005). It should be noted, however, that other studies have either failed to confirm an association with DAT1 genotype or have not supported the direction of the association reported above (Michelhaugh et al., 2001; Mill et al., 2002; Miller and Madras, 2002). Nevertheless, based upon past cognitive genetic data (Bellgrove et al., 2005, 2007, 2008, 2009; Newman et al., 2012; Zozulinsky et al., 2014) and that reported herein, it is plausible that the 10-repeat DAT1 allele is associated with relatively lower available dopamine in the right hemisphere, which would promote a rightward perceptual bias. Indeed, some evidence in healthy subjects suggests that striatal DAT binding might be higher in the right compared with left hemisphere in healthy subjects (Laakso et al., 2000). Conversely, if fewer copies of the 10-repeat DAT1 allele are associated with relatively higher available dopamine in the right hemisphere, then this would promote a leftward perceptual bias.

Although we confirm here an interaction between DAT1 genotype and a spatial bias to lateralized stimuli (see Omnibus taskcomponent analyses, DAT1 by stimulus laterality interaction), the results of the current study, however, go beyond any simple perceptual bias account. Instead, we find that allelic variation in the DAT1 gene is linked to a specific lateralized attentional mechanism. Those individuals not in possession of the 10-repeat DAT1 allele displayed an enhanced ability to suppress distracting information in the left hemifield compared with those with either one or two copies of this allele. We propose that individuals with- out the 10-repeat DAT1 allele have higher levels of synaptic dopamine occasioned by lower DAT reuptake. We further suggest that the DAT1 gene may influence attentional selection in part because of its varying effects on the development of frontoparietal attention networks that are dominant in the right hemisphere. This suggestion is supported both by the existence of dopamine projections to the frontal and parietal cortices in addition to the well documented projections to striatum, and by high densities of DAT-immunoreactive axons in the parietal lobe (Lewis et al., 2001). Thus we argue that, although perceptual biases linked to DAT1 genotype are consistent with asymmetric modulation of striatal dopamine, the specific enhancement of attentional selection seen here may be due to an effect of DAT1 genotype on dopamine modulation of frontoparietal attention networks ( $\mathrm{Pe}$ tersen and Posner, 2012). This suggestion should now be confirmed using DAT1 genotype-controlled studies and either fMRI or PET, in combination with behavioral measures, such as those employed here.

Although participants were instructed to maintain fixation across all tasks, it is possible that random fixation breaks occurred in the current study, and it would be desirable to use eye-tracking in the future. However, since target display times for the spatial competition and spatial cueing tasks were brief $(150 \mathrm{~ms})$, it is unlikely that sufficient time was available for systematic eye movements to be made to the lateralized targets. Thus, it is highly unlikely that eye movements could account for the relationship between DAT1 genotype and lateralized attentional selection.

In summary, compared with individuals carrying one or two copies of the 10-repeat DAT1 allele, individuals without this allele demonstrated an immunity to increased distracters presented in the left hemifield, suggesting they possess an enhanced attentional ability to suppress task-irrelevant stimuli within the left hemifield.

\section{Notes}

Supplemental material for this article is available at http://figshare. com/articles/Supplementary_Information_for/1032601. The crucial effects from the results section (Fig. $2 A, B$ ) were analyzed without the use of covariates. The interaction of DAT1 genotype, Distractor Hemifield, and Set Size are plotted separately for unilateral and bilateral display types. Figures depict absolute RT values for the spatial competition, spatial cueing, and flanker tasks, as opposed to the set-size, reorienting, and incongruence effects (respectively), for the tasks in the main article. This material has not been peer reviewed.

\section{References}

Bellgrove MA, Hawi Z, Kirley A, Fitzgerald M, Gill M, Robertson IH (2005) Association between dopamine transporter (DAT1) genotype, left-sided inattention, and an enhanced response to methylphenidate in attentiondeficit hyperactivity disorder. Neuropsychopharmacology 30:22902297. CrossRef Medline

Bellgrove MA, Chambers CD, Johnson KA, Daibhis A, Daly M, Hawi Z, Lambert D, Gill M, Robertson IH (2007) Dopaminergic genotype biases spatial attention in healthy children. Mol Psychiatry 12:786-792. CrossRef Medline

Bellgrove MA, Barry E, Johnson KA, Cox M, Daibhis A, Daly M, Hawi Z, Lambert D, Fitzgerald M, McNicholas F, Robertson IH, Gill M, Kirley A, Dáibhis A (2008) Spatial attentional bias as a marker of genetic risk, symptom severity, and stimulant response in ADHD. Neuropsychopharmacology 33:2536-2545. CrossRef Medline

Bellgrove MA, Johnson KA, Barry E, Mulligan A, Hawi Z, Gill M, Robertson I, Chambers CD (2009) Dopaminergic haplotype as a predictor of spatial inattention in children with attention-deficit/hyperactivity disorder. Arch Gen Psychiatry 66:1135-1142. CrossRef Medline

Brand A, Bradley MT (2012) Assessing the effects of technical variance on the statistical outcomes of web experiments measuring response times. Soc Sci Comput Rev 30:350-357. CrossRef 
Brookes KJ, Mill J, Guindalini C, Curran S, Xu X, Knight J, Chen CK, Huang YS, Sethna V, Taylor E, Chen W, Breen G, Asherson P (2006) A common haplotype of the dopamine transporter gene associated with attention-deficit/hyperactivity disorder and interacting with maternal use of alcohol during pregnancy. Arch Gen Psychiatry 63:74-81. CrossRef Medline

Brookes KJ, Neale BM, Sugden K, Khan N, Asherson P, D’Souza UM (2007) Relationship between VNTR polymorphisms of the human dopamine transporter gene and expression in post-mortem midbrain tissue. Am J Med Genet B Neuropsychiatr Genet 144B:1070-1078. CrossRef Medline

Cheon KA, Ryu YH, Kim JW, Cho DY (2005) The homozygosity for 10repeat allele at dopamine transporter gene and dopamine transporter density in Korean children with attention deficit hyperactivity disorder: relating to treatment response to methylphenidate. Eur Neuropsychopharmacol 15:95-101. CrossRef Medline

Clark CR, Geffen GM, Geffen LB (1989) Catecholamines and the covert orientation of attention in humans. Neuropsychologia 27:131-139. CrossRef Medline

Cook EH Jr, Stein MA, Krasowski MD, Cox NJ, Olkon DM, Kieffer JE, Leventhal BL (1995) Association of attention-deficit disorder and the dopamine transporter gene. Am J Hum Genet 56:993-998. Medline

Cornish KM, Manly T, Savage R, Swanson J, Morisano D, Butler N, Grant C, Cross G, Bentley L, Hollis CP (2005) Association of the dopamine transporter (DAT1) 10/10-repeat genotype with ADHD symptoms and response inhibition in a general population sample. Mol Psychiatry 10:686-698. CrossRef Medline

Coull JT, Nobre AC, Frith CD (2001) The noradrenergic $\alpha 2$ agonist clonidine modulates behavioural and neuroanatomical correlates of human attentional orienting and alerting. Cereb Cortex 11:73-84. CrossRef Medline

Damian MF (2010) Does variability in human performance outweigh imprecision in response devices such as computer keyboards? Behav Res Methods 42:205-211. CrossRef Medline

Driver J, Mattingley JB (1998) Parietal neglect and visual awareness. Nat Neurosci 1:17-22. CrossRef Medline

Eriksen CW, St James JD (1986) Visual attention within and around the field of focal attention: a zoom lens model. Percept Psychophys 40:225240. CrossRef Medline

Eriksen CW, Yeh YY (1985) Allocation of attention in the visual field. J Exp Psychol Hum Percept Perform 11:583-597. CrossRef Medline

Fleet WS, Valenstein E, Watson RT, Heilman KM (1987) Dopamine agonist therapy for neglect in humans. Neurology 37:1765-1770. CrossRef Medline

Fuke S, Suo S, Takahashi N, Koike H, Sasagawa N, Ishiura S (2001) The VNTR polymorphism of the human dopamine transporter (DAT1) gene affects gene expression. Pharmacogenomics J 1:152-156. CrossRef Medline

Glick SD, Shapiro RM (1985) Functional and neurochemical mechanisms of cerebral lateralization in rats. Cereb Lateralization Nonhum Species:157-183.

Gordon I, Rehavi M, Mintz M (1994) Bilateral imbalance in striatal DAuptake controls rotation behavior. Brain Res 646:207-210. CrossRef Medline

Gorgoraptis N, Mah YH, Machner B, Singh-Curry V, Malhotra P, HadjiMichael M, Cohen D, Simister R, Nair A, Kulinskaya E, Ward N, Greenwood R, Husain M (2012) The effects of the dopamine agonist rotigotine on hemispatial neglect following stroke. Brain 135:2478-2491. CrossRef Medline

Grujic Z, Mapstone M, Gitelman DR, Johnson N, Weintraub S, Hays A, Kwasnica C, Harvey R, Mesulam MM (1998) Dopamine agonists reorient visual exploration away from the neglected hemispace. Neurology 51:1395-1398. CrossRef Medline

Guindalini C, Howard M, Haddley K, Laranjeira R, Collier D, Ammar N, Craig I, O'Gara C, Bubb VJ, Greenwood T, Kelsoe J, Asherson P, Murray RM, Castelo A, Quinn JP, Vallada H, Breen G (2006) A dopamine transporter gene functional variant associated with cocaine abuse in a Brazilian sample. Proc Natl Acad Sci U S A 103:4552-4557. CrossRef Medline

Heinz A, Goldman D, Jones DW, Palmour R, Hommer D, Gorey JG, Lee KS, Linnoila M, Weinberger DR (2000) Genotype influences in vivo dopamine transporter availability in human striatum. Neuropsychopharmacology 22:133-139. CrossRef Medline
Iversen SD (1984) Behavioural effects of manipulation of basal ganglia neurotransmitters. Ciba Found Symp 107:183-200. Medline

Laakso A, Vilkman H, Alakare B, Haaparanta M, Bergman J, Solin O, Peurasaari J, Räkköläinen V, Syvälahti E, Hietala J (2000) Striatal dopamine transporter binding in neuroleptic-naive patients with schizophrenia studied with positron emission tomography. Am J Psychiatry 157:269_ 271. CrossRef Medline

Lewis DA, Melchitzky DS, Sesack SR, Whitehead RE, Auh S, Sampson A (2001) Dopamine transporter immunoreactivity in monkey cerebral cortex: regional, laminar, and ultrastructural localization. J Comp Neurol 432:119-136. CrossRef Medline

Maruff P, Hay D, Malone V, Currie J (1995) Asymmetries in the covert orienting of visual spatial attention in schizophrenia. Neuropsychologia 33:1205-1223. CrossRef Medline

Michelhaugh SK, Fiskerstrand C, Lovejoy E, Bannon MJ, Quinn JP (2001) The dopamine transporter gene (SLC6A3) variable number of tandem repeats domain enhances transcription in dopamine neurons. J Neurochem 79:1033-1038. Medline

Mill J, Asherson P, Browes C, D’Souza U, Craig I (2002) Expression of the dopamine transporter gene is regulated by the $3^{\prime}$ UTR VNTR: evidence from brain and lymphocytes using quantitative RT-PCR. Am J Med Genet 114:975-979. CrossRef Medline

Miller GM, Madras BK (2002) Polymorphisms in the 3'-untranslated region of human and monkey dopamine transporter genes affect reporter gene expression. Mol Psychiatry 7:44-55. CrossRef Medline

Newman DP, O'Connell RG, Nathan PJ, Bellgrove MA (2012) Dopamine transporter genotype predicts attentional asymmetry in healthy adults. Neuropsychologia 50:2823-2829. CrossRef Medline

Petersen SE, Posner MI (2012) The attention system of the human brain: 20 years after. Annu Rev Neurosci 35:73-89. CrossRef Medline

Posner MI, Walker JA, Friedrich FJ, Rafal RD (1984) Effects of parietal injury on covert orienting of attention. J Neurosci 4:1863-1874. Medline

Sheppard DM, Bradshaw JL, Mattingley JB, Lee P (1999) Effects of stimulant medication on the lateralisation of line bisection judgements of children with attention deficit hyperactivity disorder. J Neurol Neurosurg Psychiatry 66:57-63. CrossRef Medline

Silk TJ, Newman DP, Eramudugolla R, Vance A, Bellgrove MA (2014) Influence of methylphenidate on spatial attention asymmetry in adolescents with attention deficit hyperactivity disorder (ADHD): preliminary findings. Neuropsychologia 56:178-183. CrossRef Medline

Simmons JP, Nelson LD, Simonsohn U (2011) False-positive psychology: undisclosed flexibility in data collection and analysis allows presenting anything as significant. Psychol Sci 22:1359-1366. CrossRef Medline

Stephens M, Donnelly P (2003) A comparison of Bayesian methods for haplotype reconstruction from population genotype data. Am J Hum Genet 73:1162-1169. CrossRef Medline

Stephens M, Smith NJ, Donnelly P (2001) A new statistical method for haplotype reconstruction from population data. Am J Hum Genet 68:978989. CrossRef Medline

Tomer R, Slagter HA, Christian BT, Fox AS, King CR, Murali D, Davidson RJ (2012) Dopamine asymmetries predict orienting bias in healthy individuals. Cereb Cortex 23:2899-2904. CrossRef Medline

VanNess SH, Owens MJ, Kilts CD (2005) The variable number of tandem repeats element in DAT1 regulates in vitro dopamine transporter density. BMC Genet 6:55. CrossRef Medline

Voytko ML, Olton DS, Richardson RT, Gorman LK, Tobin JR, Price DL (1994) Basal forebrain lesions in monkeys disrupt attention but not learning and memory. J Neurosci 14:167-186. Medline

Ward NM, Brown VJ (1996) Covert orienting of attention in the rat and the role of striatal dopamine. J Neurosci 16:3082-3088. Medline

Witte EA, Davidson MC, Marrocco RT (1997) Effects of altering brain cholinergic activity on covert orienting of attention: comparison of monkey and human performance. Psychopharmacology (Berl) 132:324-334. CrossRef Medline

Yang Q, Kapoula Z, Debay E, Coubard O, Orssaud C, Samson M (2006) Prolongation of latency of horizontal saccades in elderly is distance and task specific. Vis Res 46:751-759. CrossRef Medline

Zozulinsky P, Greenbaum L, Brande-Eilat N, Braun Y, Shalev I, Tomer R (2014) Dopamine system genes are associated with orienting bias among healthy individuals. Neuropsychologia 62:48-54. CrossRef Medline 